\title{
Performance Evaluation of Empirical Path Loss Models for a Linear Wireless Sensor Network Deployment in Suburban and Rural Environments
}

\section{Yaser Dalveren ${ }^{1}$ and Ali Kara ${ }^{2}$ (iD}

${ }^{1}$ Atilim University, Department of Avionics, Ankara, Turkey

${ }^{2}$ Atilim University, Department of Electrical and Electronics Engineering, Ankara, Turkey

\section{ABSTRACT}

$\mathrm{T}$ his article presents a preliminary propagation study on the accuracy of empirical path loss models for efficient planning and deployment of a linear wireless sensor network (LWSN) based on long range (LoRa) enabled sensor nodes in suburban and rural environments. Real-world deployment of such network requires accurate path loss modelling to estimate the network coverage and performance. Although several models have been studied in the literature to predict the path loss for LoRa links, the assessment of empirical path loss models within the context of low-height peer to peer configured system has not been provided yet. Therefore, this study aims at providing a performance evaluation of well-known empirical path loss models including the Log-distance, Okumura, Hata, and COST-231 Hata model in a peer to peer configured system where the sensor nodes are deployed at the same low heights. To this end, firstly, measurement campaigns are carried out in suburban and rural environments by utilizing LoRa enabled sensor nodes operating at $868 \mathrm{MHz}$ band. The measured received signal strength values are then compared with the predicted values to assess the prediction accuracy of the models. The results achieved from this study show that the Okumura model has higher accuracy.

\section{Keywords:}

Propagation models; LoRa; Path loss; Suburban; Rural.

\author{
Article History: \\ Received: 2020/07/10 \\ Accepted: 2020/11/16 \\ Online: 2020/12/31
}

Correspondence to: Yaser Dalveren, Atilim University, Avionics, 06830, Ankara, TURKEY

E-Mail:yaser.dalveren@atilim.edu.tr Phone: +903125868320

\section{INTRODUCTION}

Tn the past few years, Wireless Sensor Networks L(WSNs) have been evolving in many different aspects such as agricultural, industrial, and surveillance. In order to collect sensor data, the wireless nodes are deployed in an area with a specific network topology. Although there are various network topologies to deploy WSNs, the geographic conditions of the given area and the purpose of the application have still vital importance to decide the structure of network topology. Especially, when linear structures like pipelines, railways, roads or tunnels need to be monitored, WSN are formed as linear network topology which is known as linear-WSN (LWSN) [1].

In a typical LWSN, the sensor nodes are communicated within a hierarchical order. The hierarchical structure requires the single-hop communication model between the transmitting and receiving nodes. Generally, for an outdoor deployment, the communication range between these nodes is desired to be kept as high as possible. For this reason, it is necessary to use proper radio transceiver that enables long-range communication distances. During the last years, the LoRa (Long Range) which is one of the low-power wide-area networks (LPWAN) technologies operating in the licensefree ISM frequency band technology offers important advantages in communication range [2]. Its efficiency has been experimentally proven by various studies [312]. Recently, it has been also adapted to several LWSNbased applications [13-15].

On the other hand, real-world deployment of LWSNs requires an accurate prediction of network coverage and performance. In this context, a great understanding of the propagation impairments that affect the propagation links is strictly needed for designing such networks. One of the impairments adversely affecting the links is already known as path loss which describes the variation in the received signal power based on the distance between the receiving and transmitting nodes. Path loss also depends on the area or environment where the network is deployed. In this context, the environ- 
mental conditions (terrain profile or topography) in terms of buildings, mountains, and hills that directly impact on path loss must be taken into account. Therefore, real-world deployment of LSWNs require accurate path loss modelling to estimate the network coverage.

Even though there are several path loss models that can be applicable to outdoor links for various bands, there has been no specific model to predict the path loss for LoRa links until now. However, many empirical path loss models have been studied in the literature, most of which consider the urban environment [8-10, 16-20]. Only few studies have evaluated empirical path loss models for suburban and/or rural environments $[8,9,19,20]$. It is worth noting that these studies are based on base station/gateway to mobile terminal configuration where the transmitter is deployed higher than the receivers. As far as we know, the assessment of well-known empirical path loss models within the context of low-height peer to peer configured system for LoRa links has not been provided yet.

This study presents preliminary propagation study for accurate deployment of a LWSN-based monitoring system proposed in [15]. The main purpose is to provide performance evaluation of well-known empirical path loss models, namely the Log-distance, Okumura, Hata, and COST-231 Hata model in a low-height peer to peer configured system. In this context, the predicted received signal strength indicator (RSSI) values are compared with the propagation measurements carried out in suburban and rural areas at 868 $\mathrm{MHz}$ using LoRa enabled sensor nodes in order to evaluate the prediction accuracy of the models. The comparison results show that the Okumura model has higher prediction accuracy when compared to other models. Thus, to the best of our knowledge, this is the first study in the literature that provides the assessment of well-known empirical path loss models within the context of low-height peer to peer configured system for LoRa links. Besides, it is believed that the initial results achieved from in this study offer useful insights for efficient planning and deployment of LWSNs

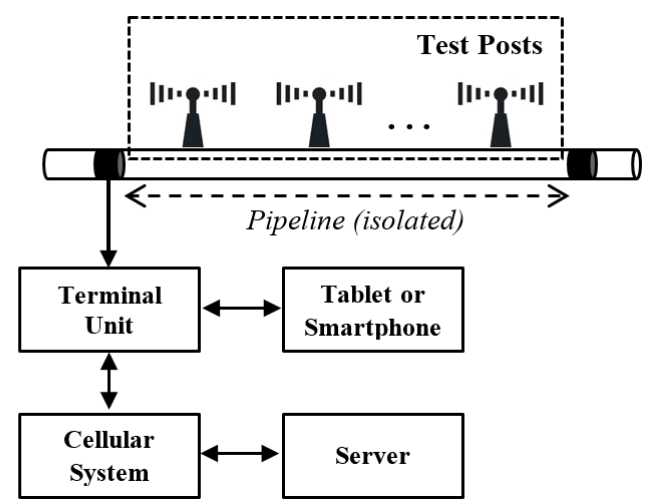

Figure 1. The LWSN-based Monitoring System [15]. based on LoRa enabled sensor nodes or transceivers in suburban and rural environments such as [15].

The article is organized as follows. In the following section, LWSN-based monitoring system is overviewed. The details about the field measurements are then presented in Section 3. Next, the path loss models investigated under this study are described in Section 4. In Section 5, the comparison results are provided and discussed. Conclusions are given in the last section.

\section{RELATED WORK}

The LWSN-based system in [15] is proposed as an alternative to traditional cathodic protection (CP) monitoring of oil and natural gas pipeline systems. Contrary to traditional CP monitoring systems, as a novelty, the proposed system is designed to be used in extreme environments (deep valleys, desserts, etc.) where the communication infrastructure is limited or unavailable. The main structure of the system is illustrated in Fig. 1. As shown in the figure, the system consists test posts (TPs), each of which have a sensor node connected to the measurement unit. Here, low-bit sensor nodes with low-antenna height are used to provide bidirectional links. In this way, each TP is able to transmit the collected data through the neighbour TPs in a sequence.

In this system, the sensor nodes typically include LoRa module to achieve the maximum reasonable communication range. Evidently, a large-scale deployment of this system requires accurate characterization of the propagation channel. As mentioned previously, most of the existing works on the performance of LoRa coverage use the gateways/transmitters located much higher than the mobile terminals/ receivers. However, when the system architecture shown in Fig. 1 is concerned, it is necessary to provide accurate models for peer to peer configuration where the transmitter and the receiver are located at the same low heights. Otherwise, inaccurate models will result in poor decision making during large-scale deployment of the LWSN system. Accordingly, for an accurate deployment of the given sensor network, the effects of the distance between the sensor nodes and the environmental conditions on the LoRa links in suburban and rural environments should be examined.

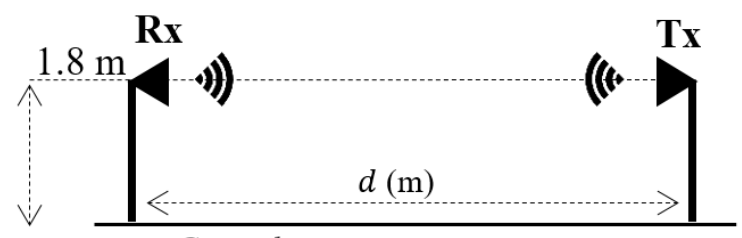

Ground

Figure 2. Link configuration of the measurement campaign. 


\section{MEASUREMENT SETUP}

In order to evaluate the accuracy of the well-known empirical path loss models for suburban and urban environments, a set of propagation measurements are taken by the LoRa enabled sensor nodes. Fig. 2 shows the conceptual overview of the measurement setup.

The measurement setup consists of a transmitter node $(\mathrm{Tx})$ and a receiver node $(\mathrm{Rx})$. For receiving purposes, the sensor node shown in Fig. 3 is used. It comprises a microstrip antenna with $1 \mathrm{dBi}$ gain [21] and a LoRa (SX1276) module which is stacked on Arduino Mega board. For transmitting purposes, the sensor node which is designed to be used in the LWSN-based system discussed in the previous section is used (Fig. 4). It comprises a LoRa module, two directive antennas, and a RF splitter for coupling the LoRa output to the antenna ports. In fact, it is designed to use two directive antennas placed back-to-back to provide bidirectional links in a real deployment. However, for this study, one of the ports of the Tx was disabled by connecting a standard flat antenna to provide peer to peer configuration. An iden-



Figure 3. The receiver node ( $\mathrm{Rx})$ used for path loss measurements.

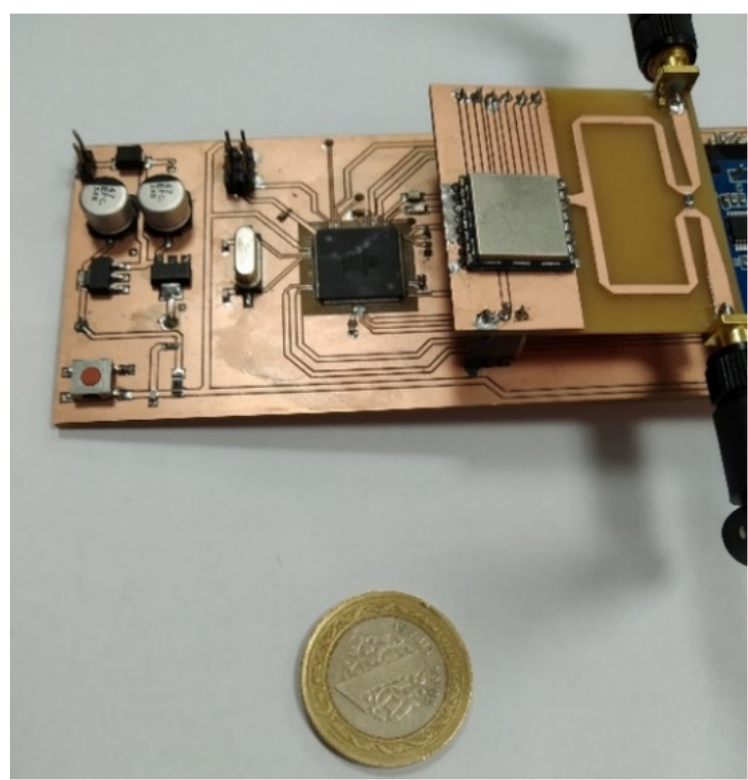

Figure 4. The transmitter node (Tx) used for path loss measurements.
Table 1. LoRa settings and the configurations used for the Tx and Rx.

\begin{tabular}{cc}
\hline \multicolumn{2}{c}{ Parameters for the Measurements } \\
\hline Spreading factor & 7 \\
Bandwidth & $125 \mathrm{kHz}$ \\
Coding rate & $4 / 5$ \\
Output power & $17 \mathrm{~dB}$ \\
Center frequency & $868 \mathrm{MHz}$ \\
Payload length & $8 \mathrm{bytes}$ \\
Preamble & 6 symbols \\
\hline
\end{tabular}

tical antenna used in the Rx was then connected to the other port of the Tx. By means of $1 \mathrm{~m}$ SMA cable, the microstrip antennas of the nodes were then mounted on the top of the stands with equal heights above the ground $(1.8 \mathrm{~m})$. On the other hand, the LoRa settings and the configurations used for the Tx and the Rx are listed in Table 1.

The measurements were performed in suburban and rural environments around the Atilim University campus located in Incek, Ankara (Turkey). The suburban environment is composed of a combination of trees, weeds, and buildings with about $4 \mathrm{~m}, 10 \mathrm{~cm}$, and $7 \mathrm{~m}$ heights as shown in Fig. 5 (a). The rural environment is mainly composed of weeds with $10 \mathrm{~cm}$ height (Fig. 5 (b)).

During the measurements, the Tx was fixed at a laboratory of the Atilim University, and the Rx was moved by following a specific path over the measurement points. The propagation environments considered for the measurements is non line-of-sight (NLOS) because of no direct visibility between the Tx and Rx in the measurement points. The separation distance between the Tx and the $\mathrm{Rx}(\mathrm{d})$ varied in the range of $0.5-5 \mathrm{~km}$. At the measurement points, the Tx was configured to send 100 packets. The measurements were repeated five times to remove local propagation (multipath) effects. In total, over 40 measurements were taken at 8 measurement points in each environment. Mean RSSI values

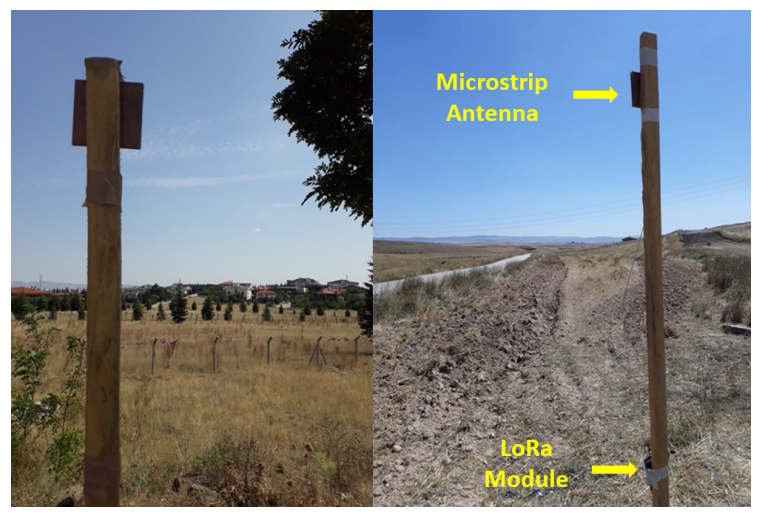


were then calculated using the recorded measurements. For LoRa (SX1276), it is useful to note that the RSSI is a measure of the incoming signal power at $\mathrm{RF}$ input port $\left(\mathrm{P}_{\mathrm{r}}\right.$, in $\left.\mathrm{dBm}\right)$ where the signal power (RssiValue) can be expressed as [22]

$$
\text { RssiValue }=-2 \cdot P_{r}+\beta
$$

where is $\beta$ is the RSSI offset (in $\mathrm{dB}$ ). The effective signal strength (in $\mathrm{dBm}$ ) is then obtained as follows

$$
R S S I=\frac{-R_{\text {ssiValue }}}{2}
$$

Besides, in order to evaluate the signal strength of the received packet, the following expression is also used

$$
\text { Packet Strength }(\mathrm{dBm})=-157+R S S I_{\text {packet }}
$$

where $R S S I_{\text {packet }}$ is an averaged of RSSIs.

Moreover, Packet Reception Ratio (PRR), which is the proportion of the number of packets successfully received to the packets transmitted in total, were calculated at each measurement point where the targeted packet reception rate (PRR) is around 95\% for the low bit rate LWSN system in [15].

\section{PATH LOSS MODELS}

To get a deeper insight of wireless channel characterization, an analysis of the propagation in terms of received power is required. Typically, the received power $\left(P_{r}\right.$, in $\mathrm{dBm})$ can be calculated as [23]

$$
P_{r}=P_{t}+G_{r}+G_{t}-L_{p}
$$

where $P_{t}$ is transmitted power (in $\mathrm{dBm}$ ), $G_{r}$ and $G_{t}$ are the antenna gains respectively of the receiver and transmitter (in $\mathrm{dB}$ ), and $L_{p}$ is the path loss (in $\mathrm{dB}$ ) which is an attenuation that is caused by the range between the receiver and the transmitter as well as the characteristics of the environment. As the $L_{p}$ significantly affects the propagation links, it needs to be predicted for an accurate design of the networks.

In the following, the empirical path loss models for rural and suburban links that can be used in the frequency band dedicated to LoRa in Europe (868 MHz) are presented.

\section{Log-distance Model}

As it is widely known, the propagation channels based on theoretical or measurement indicate that the average $P_{r}$ tends to decrease logarithmically with distance. This can be expressed by [24]

$$
L_{p}(d)=L_{p}\left(d_{0}\right)+10 n \log \left(\frac{d}{d_{0}}\right)+X_{\sigma},
$$

where $L_{p}(d)$ is the path loss (in $\mathrm{dB}$ ) for a transmitter-receiver separation distance $d$ (in meters), $L_{p}\left(d_{0}\right)$ is the reference path loss (in $\mathrm{dB}$ ) calculated using the free space path loss formulation at a given reference distance $d_{0}$ (in meters), $n$ is the path loss exponent which varies based on the propagation environment, $X_{\sigma}$ is the shadowing effect that is a zero-mean Gaussian random variable with standard deviation $\sigma$ (in $\mathrm{dB}$ ).

\section{Okumura Model}

The Okumura model, which is one of the most used models in radio propagation, is applicable for the frequency range between $150 \mathrm{MHz}$ and $1920 \mathrm{MHz}$ and the distances between $1 \mathrm{~km}$ and $100 \mathrm{~km}$. To determine the $L_{p}$, firstly, the free space path loss between the measurement points is determined. Then, the value of the median attenuation relative to free space and correction factors are added. It can be expressed as [25]

$$
L_{p}=L_{f}+A_{\mu}-G\left(h_{t}\right)-G\left(h_{r}\right)-G_{A}
$$

where $L_{f}$ is the free space path loss, $A_{\mu}$ is the median attenuation relative to free space, $G(h)$ is the transmitter or base station antenna height gain factor, $G\left(h_{r}\right)$ is the receiver or mobile antenna height gain factor, and $G_{A}$ is the gain that varies according to the environment. The values of $A_{\mu}$ and $G_{A}$ can be reached from the Okumura's empirical plots $[24,25]$. The $G\left(h_{t}\right)$ and $G\left(h_{r}\right)$ can be calculated from the following expressions

$$
\begin{aligned}
& G\left(h_{t}\right)=20 \log \left(\frac{h_{t}}{200}\right) \text { for } 1000 m>h_{t}>30 m, \\
& G\left(h_{r}\right)=10 \log \left(\frac{h_{r}}{3}\right) \text { for } h_{r} \leq 3 m, \\
& G\left(h_{r}\right)=20 \log \left(\frac{h_{r}}{3}\right) \text { for } 10 m>h_{r}>3 m .
\end{aligned}
$$

\section{Hata Model}

The Hata model is another widely used model to predict path loss in radio propagation. It formulates the Okumura's empirical plots for various parameters. It is applicable for the frequency range between $150 \mathrm{MHz}$ and $1500 \mathrm{MHz}$ and the distances between $1 \mathrm{~km}$ and $20 \mathrm{~km}$. This model is presented to calculate the $L_{p}$ in urban environments, however, it incorporates path loss factors and correction factor for the applications in suburban and rural environments. The path loss in urban environments $\left(L_{p, \text { urban }}\right)$ is expressed as [26]

$$
\begin{aligned}
L_{p, \text { urban }}= & 69.55+26.16 \log f_{c}-13.82 \log h_{t}-\alpha_{h_{r}} \\
& +\left(44.9-6.55 \log h_{t}\right) \log d
\end{aligned}
$$

where $f_{c}$ is the frequency (in $\mathrm{MHz}$ ), $h_{t}$ is the transmitter or base station antenna height ranging from $30 \mathrm{~m}$ to $200 \mathrm{~m}$, $h_{r}$ is the receiver or mobile antenna height ranging from $1 \mathrm{~m}$ to $10 \mathrm{~m}, \mathrm{~d}$ is defined in (5), and $\alpha_{h_{r}}$ is the correction factor for the receiver antenna height (in $\mathrm{dB}$ ). It is given 


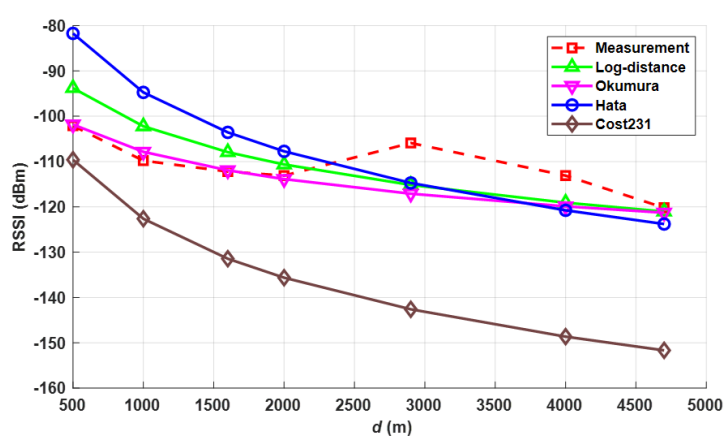

Figure 6. Comparison of the measured and simulated data (rural).

for a small to medium sized city by

$$
\alpha_{h_{r}}=\left(1.1 \log f_{c}-0.7\right) h_{r}-\left(1.56 \log f_{c}-0.8\right) .
$$

Then, the path loss in a suburban environment is obtained by

$$
L_{p, \text { suburban }}=L_{p, \text { urban }}-2\left[\log \left(f_{c} / 28\right)\right]^{2}-5.4,
$$

and the path loss in a rural environment is obtained by

$$
L_{p, \text { rural }}=L_{p, \text { urban }}-4.78 \log f_{c}^{2}+18.33 \log f_{c}-40.98 \text {. }
$$

\section{COST-231 Model}

This model is developed as an extended version of the Hata model which is designed to be used in the frequency band ranging from $500 \mathrm{MHz}$ to $2000 \mathrm{MHz}$. It is widely used to predict path loss due to its simplicity. The model can be expressed as follows [27]

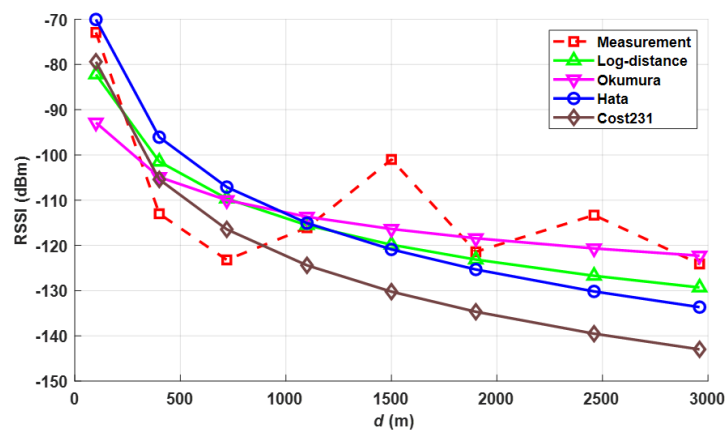

Figure 7. Comparison of the measured and simulated data (suburban).

$$
\begin{aligned}
L_{p}= & 46.3+33.9 \log f_{c}-13.82 \log h_{t}-\alpha_{h_{r}} \\
& +\left(44.9-6.55 \log h_{t}\right) \log d+C_{m}
\end{aligned}
$$

where the parameters $f_{c}, h_{t}, h_{r}, \alpha_{h_{r}}$, and $d$ are defined in (10) while the correction factor $\left(C_{m}\right)$ is defined as $0 \mathrm{~dB}$ for suburban or rural environments.

\section{RESULTS AND ANALYSIS}

In order to assess the prediction accuracy of the empirical path loss models under this study, the RSSI measurements are compared with the simulation data obtained using the models. Fig. 6 and Fig. 7 show comparison between the measured and the models in rural and suburban link, respectively. Table 2 and Table 3 also present the

\begin{tabular}{|c|c|c|c|c|c|c|c|}
\hline \multirow{2}{*}{ Method } & \multicolumn{7}{|c|}{ Distance (m) } \\
\hline & 500 & 1000 & 1600 & 2000 & 2900 & 4000 & 4700 \\
\hline Measurement & -102.1 & -109.8 & -112.2 & -113.1 & -105.9 & -113.1 & -120.2 \\
\hline Log-distance model & -93.8 & -102.2 & -107.9 & -110.6 & -115.2 & -119.1 & -121 \\
\hline Okumura model & -101.8 & -107.9 & -111.9 & -113.9 & -117.1 & -119.9 & -121.3 \\
\hline Hata model & -81.71 & -94.7 & -103.5 & -107.7 & -114.7 & -120.7 & -123.8 \\
\hline COST-231 Hata model & -109.6 & -122.6 & -131.4 & -135.6 & -142.6 & -148.6 & -151.7 \\
\hline
\end{tabular}
corresponding values. It should be noted that the path loss exponent $\mathrm{n}$ is determined as $6 \mathrm{~dB}$ while the parame-

\begin{tabular}{|c|c|c|c|c|c|c|c|c|}
\hline \multirow{2}{*}{ Method } & \multicolumn{8}{|c|}{ Distance $(m)$} \\
\hline & 100 & 400 & 720 & 1100 & 1500 & 1900 & 2460 & 2960 \\
\hline Measurement & -72.9 & -113 & -123.2 & -116.1 & -101 & -121.4 & $-113 \cdot 3$ & -124.1 \\
\hline Log-distance model & -82.2 & -101.5 & -109.6 & $-115 \cdot 5$ & -119.8 & -123.1 & -126.7 & $-129 \cdot 3$ \\
\hline Okumura model & -92.8 & -104.9 & -110 & -113.7 & -116.4 & -118.4 & -120.7 & -122.3 \\
\hline Hata model & -70 & -96.1 & -107.1 & -115.1 & -120.9 & $-125 \cdot 3$ & -130.2 & -133.6 \\
\hline COST-231 Hata model & $-79 \cdot 4$ & -105.4 & -116.5 & $-124 \cdot 4$ & -130.2 & -134.7 & -139.5 & -142.9 \\
\hline
\end{tabular}
ter $X_{\sigma}$ is determined as 2.8 and 3.2 for rural and suburban

Table 2. Mean values of the RSSI measurement and the simulation data in rural link (dBm).

Table 3. Mean values of the RSSI measurement and the simulation data in suburban link (dBm). 
link, respectively [24]. Besides, for the COST-231 Hata model, the parameter $C_{m}$ is defined as $0 \mathrm{~dB}$ for both rural and suburban link [24, 27]. Moreover, for the Okumura model, the parameter $A_{\mu}$ is defined as $19 \mathrm{~dB}$ while the parameter $G_{A}$ is defined as $26.5 \mathrm{~dB}$ and $21.5 \mathrm{~dB}$ for rural and suburban link, respectively $[24,25]$.

At first glance, as can be seen from the results achieved for the rural link, the measured RSSI values agree well with the Log-distance and Okumura model simulation, and mostly agree with the Hata model simulation. However, the predicted values by the COST-231 Hata differ from the RSSI values. In rural link, it is worth noting that the PRR substantially reduced beyond the measurement point at $4.7 \mathrm{~km}$ (above 95\%). Beyond that point, as the percentage of losses dramatically increased, it is determined as the maximum achievable communication distance.

On the other hand, the results achieved for the suburban link show that the predicted values obtained by the Log-distance, Okumura, and Hata model relatively agree with the RSSI values while the predicted values obtained by the COST-231 Hata model again differ from the RSSI values. Here, based on the PRRs, the maximum achievable distance is also found to be $2.7 \mathrm{~km}$ for suburban link.

The prediction errors of the path loss models are also calculated to evaluate their performance in detail. To do this, the error statistics such as the mean absolute error $\left(\Delta_{e}\right)$, the standard deviation of the prediction error $(\sigma)$, and the mean prediction error $\left(\mu_{e}\right)$ are used [16]. The metric $\mu_{e}$ can be calculated by

$$
\mu_{e}=1 / N \sum_{i=1}^{N} \Delta_{R S S I}
$$

where $N$ denotes the number of samples, and $\Delta_{R S S I}$ is the difference between the predicted data $\left(R S S I_{p}\right)$ and measured data $\left(R S S I_{m}\right)$ which can be obtained by

$$
\Delta_{R S S I}=R S S I_{p}-R S S I_{m} .
$$

The metric $\Delta_{e}$ and $\sigma_{e}$ can be then calculated by

$$
\begin{aligned}
& \Delta_{e}=1 / N \sum_{i=1}^{N}\left|\Delta_{R S S I}\right|, \\
& \text { and } \\
& \sigma=\sqrt{1 / N \sum_{i=1}^{N}\left(\Delta_{R S S I}-\Delta_{e}\right)^{2}} .
\end{aligned}
$$

Thus, the prediction errors of the models are listed in Table 4 and Table 5 for rural and suburban link, respectively. Here, a negative or positive value of the $\mu_{e}$ metric indicates that the model underestimates or overestimates the received signal power. Moreover, $\Delta_{e}$ corresponds to the prediction accuracy of the models. In this context, the small value of $\Delta_{e}$ indicates that the model has higher prediction accuracy.
Table 4. Prediction errors of the models (rural).

\begin{tabular}{ccccc}
\hline $\begin{array}{c}\text { Error } \\
\text { Metric }\end{array}$ & $\begin{array}{c}\text { Log- } \\
\text { distance }\end{array}$ & Okumura & Hata & $\begin{array}{c}\text { COST-231 } \\
\text { Hata }\end{array}$ \\
\hline$\mu_{e}$ & 0,9 & $-2,5$ & 4,2 & $-23,7$ \\
$\Delta_{e}$ & 5,5 & 3,2 & 9,9 & 23,7 \\
$\sigma_{e}$ & 7,7 & 7,2 & 12 & 48,5 \\
\hline
\end{tabular}

Table 5. Prediction errors of the models (suburban).

\begin{tabular}{ccccc}
\hline $\begin{array}{c}\text { Error } \\
\text { Metric }\end{array}$ & $\begin{array}{c}\text { Log- } \\
\text { distance }\end{array}$ & Okumura & Hata & $\begin{array}{c}\text { COST-231 } \\
\text { Hata }\end{array}$ \\
\hline$\mu_{e}$ & $-2,9$ & $-1,7$ & $-1,8$ & -11 \\
$\Delta_{e}$ & 9,3 & 8,9 & 10,9 & 14,6 \\
$\sigma_{e}$ & 16,1 & 15,1 & 18 & 28,6 \\
\hline
\end{tabular}

Based on the results listed in Table 4, it is clear that the Logdistance and Hata model overestimate the received signal power in rural link while other models underestimate the received signal power. It is also evident that the Okumura model has higher prediction accuracy when compared to other models $\left(\Delta_{e}=3,2, \sigma_{e}=7,2\right)$. According to the results provided in Table 5 , all path loss models underestimate the received signal power in suburban link. For this case, however, the Okumura, Log-distance and Hata model behave similarly and achieve similar prediction accuracy when considering $\Delta_{e}$ and $\sigma_{e}$. Yet, the Okumura model seems to have relatively higher prediction accuracy $\left(\Delta_{e}=8,9, \sigma_{e}=15,1\right)$ than the other models. On the other hand, one of the important factors that highly affect the performance of the COST-231 Hata model is the parameter $C_{m}$. Although it is defined as $0 \mathrm{~dB}$ for both suburban and open (rural) environments in many sources in the literature, it may have lower values when the rural environment conditions are considered. However, this requires more experimental study and comprehensive analysis.

In order to assess the prediction accuracies of the models in more detail, the Cumulative Distribution Function (CDF) for a Gaussian (in $\mathrm{dB}$ ) distribution function of $\left|\Delta_{R S S I}\right|$ for all models in rural and suburban link are represented in Fig. 8 and Fig. 9, respectively. From the figures, it is clear that the COST-231 Hata model has the worst prediction accuracy in both rural and suburban link. From Fig. 8, on the other hand, it can be observed that the Okumura model achieves the best prediction accuracy where it manages to achieve $38 \%$ of the points that have an error under $5 \mathrm{dBm}$ while the Log-distance the Hata model achieve 33\% and 12\% of the points, respectively. As shown in Fig. 9, the accuracy of the models is significantly reduced where the Okumura model only manages to achieve $17 \%$ of the points that have an error under $5 \mathrm{dBm}$ while the Log-distance the Hata model achieve $10 \%$ and $16 \%$ of the points, respectively. Thus, although the Okumura model has slightly better prediction 


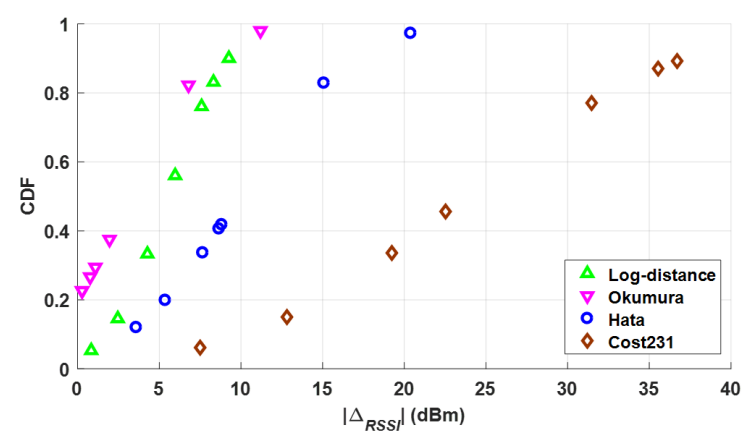

Figure 8. CDF of $\left|\Delta_{R S S I}\right|$ for all models (rural).

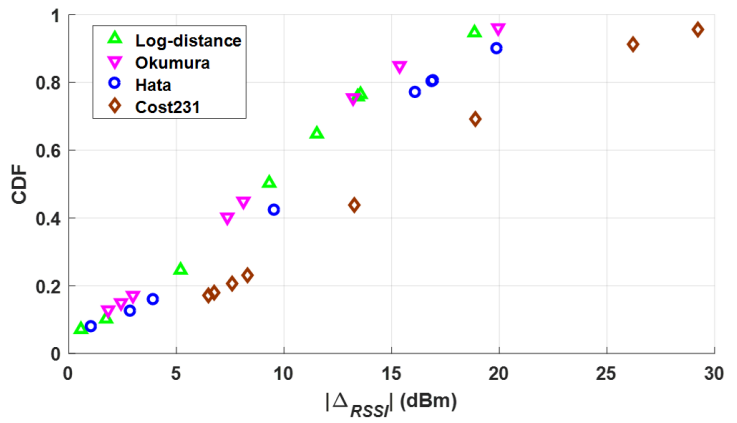

Figure 9. $\mathrm{CDF}$ of $\left|\Delta_{\text {RSSI }}\right|$ for all models (suburban).

accuracy than the Hata model, it is still necessary to enrich the number of measurements in order to achieve certain performance results in suburban link. This, in fact, constitutes a part of future works as discussed in the following section.

\section{CONCLUSION AND FUTURE WORK}

In this work, an assessment of well-known empirical path loss models (the Log-distance, Okumura, Hata, and COST-231 Hata model) within a context of low-height peer to peer configured system for LoRa links in suburban and rural environments is provided. The accuracy of path loss models is evaluated by comparing the predicted RSSI values with the measurements conducted at 868 $\mathrm{MHz}$ in suburban and rural environments around the Atilim University campus, Ankara. The results show that the Okumura model provides higher prediction accuracy while the COST-231 Hata model has lower accuracy in both suburban and rural environments.

This study constitutes as a preliminary propagation study of an ongoing project on the development of LWSNbased CP monitoring of oil and natural gas pipeline systems. Obviously, the number of the collected data from the measurements is relatively insufficient to provide an exact result especially in suburban links. For this reason, in the near future, the current work is expected to be enhanced by increasing the data size with more field tests and measurements. Particularly, the path loss effects of LWSN under development will be characterized by conducting measurement campaigns using LoRa enabled sensor nodes at different heights from the ground (in the range of $0.25 \mathrm{~m}$ to $2 \mathrm{~m}$ ) in various types of environments. But still, we believe that even this initial study would offer useful insights for many researchers who work on the planning and deployment of LWSNs based on LoRa enabled sensor nodes in suburban and rural environments.

\section{References}

1. Jawhar I, Mohamed N, Agrawal DP. Linear wireless sensor networks: Classification and applications. Journal of Network and Computer Applications 34(5) (2011) 1671-1682. doi: 10.1016/j. jnca.2011.05.006.

2. Centenaro M, Vangelista L, Zanella A, Zorzi M. Long-range communications in unlicensed bands: The rising stars in the IoT and smart city scenarios. IEEE Wireless Communications 23(5) (2016) 60-67. doi: 10.1109/MWC.2016.7721743.

3. Neumann P, Montavont J, Noël T. Indoor deployment of lowpower wide area networks (LPWAN): A LoRaWAN case study. Paper presented at the IEEE 12th International Conference on Wireless and Mobile Computing, Networking and Communications (WiMob), New York, 1-8, 2016. doi: 10.1109/WiMOB.2016.7763213.

4. Petajajarvi J, Mikhaylov K, Yasmin R, Hamalainen M, Iinatti J. Evaluation of LoRa LPWAN technology for indoor remote health and wellbeing monitoring. International Journal of Wireless Information Networks 24(2) (2017) 153-165. doi: s10776-017-03418.

5. Gregora L, Vojtech L, Neruda M. Indoor signal propagation of LoRa technology. Paper presented at the 17th International Conference on Mechatronics - Mechatronika (ME), Prague, 1-4, 2016.

6. Nolan KE, Guibene W, Kelly MY. An evaluation of low power wide area network technologies for the Internet of Things. Paper presented at the International Wireless Communications and Mobile Computing Conference (IWCMC), Paphos, 439-444, 2016. doi: 10.1109/IWCMC.2016.7577098.

7. Petric T, Goessens M, Nuaymi L, Toutain L, Pelov A. Measurements, performance and analysis of LoRa FABIAN, a realworld implementation of LPWAN. Paper presented at the IEEE 27th Annual International Symposium on Personal, Indoor, and Mobile Radio Communications (PIMRC), Valencia, 1-7, 2016. doi: 10.1109/ PIMRC.2016.7794569.

8. Petajajarvi J, Mikhaylov K, Roivainen A, Hanninen T, Pettissalo M. On the coverage of LPWANs: Range evaluation and channel attenuation model for LoRa technology. Paper presented at the 14th International Conference on ITS Telecommunications (ITST), Copenhagen, 55-59, 2015. doi: 10.1109/ITST.2015.7377400.

9. Lauridsen M, Nguyen H, Vejlgaard B, Kovacs IZ, Mogensen P, Sorensen M. Coverage comparison of GPRS, NB-IoT, LoRa, and SigFox in a $7800 \mathrm{~km} 2$ area. Paper presented at the IEEE 85th Vehicular Technology Conference (VTC Spring), Sydney, 1-5, 2017. doi: 10.1109/VTCSpring.2017.8108182.

10. Sanchez-Iborra R, Sanchez-Gomez J, Ballesta-Vinas J, Cano MD, Skarmeta AF. Performance evaluation of LoRa considering scenario conditions. Sensors 18(3) (2018) 772. doi:10.3390/s18030772.

11. Iova O, Murphy AL, Picco GP, Ghiro L, Molteni D. LoRa from the city to the mountains: Exploration of hardware and environmental factors. Paper presented at the 2017 International Conference on Embedded Wireless Systems and Networks, Sweden, 317-322, 2017. 12. Del Campo G, Gomez I, Calatrava S, Martinez R, Santamaria A. 
Power distribution monitoring using LoRa: Coverage analysis in suburban areas. Paper presented at the 2018 International Conference on Embedded Wireless Systems and Networks, Madrid, 233-238, 2018

13. Abrardo A, Pozzebon A. A Multi-hop LoRa linear sensor network for the monitoring of underground environments: The case of the medieval aqueducts in Siena, Italy. Sensors 19 (2019) 402. doi: 10.3390/s19020402.

14. Abrardo A, Fort A, Landi E, Mugnaini M, Panzardi E, Pozzebon A Black powder flow monitoring in pipelines by means of multi-hop LoRa networks. Paper presented at the II Workshop on Metrology for Industry 4.0 and IoT (MetroInd4.0\&IoT), Naples, Italy, 312-316, 2019. doi: 10.1109/METROI4.2019.8792890.

15. Kara A, Imran MAA, Karadag K. Linear wireless sensor networks for cathodic protection monitoring of pipelines. Paper presented at the IEEE International Conference on Mechatronics, Robotics and Systems Engineering, Bali, 233-236, 2019. doi:10.1109/ MoRSE48060.2019.8998664.

16. Harinda E, Hosseinzadeh S, Larijani H, Gibson RM. Comparative performance analysis of empirical propagation models for LoRaWAN $868 \mathrm{MHz}$ in an urban scenario. Paper presented at the IEEE 5th World Forum on Internet of Things (WF-IoT), Limerick, Ireland, 154-159, 2019. doi: 10.1109/WF-IoT.2019.8767245.

17. Jörke P, Böcker S, Liedmann F, Wietfeld C. Urban channel models for smart city IoT-networks based on empirical measurements of LoRa-links at 433 and $868 \mathrm{MHz}$. Paper presented at the IEEE 28th Annual International Symposium on Personal, Indoor, and Mobile Radio Communications (PIMRC), Montreal, 1-6, 2017. doi: 10.1109/PIMRC.2017.8292708.

18. Paredes M, Bertoldo S, Carosso L, Lucianaz C, Marchetta E, Allegretti M, Savi P. Propagation measurements for a LoRa network in an urban environment. Journal of Electromagnetic Waves and Applications 33(15) (2019) 2022-2036. doi: 10.1080/09205071.2019.1661287.

19. Linka H, Michael R, Aliuy OG, Jonas K. Path loss models for lowpower wide-area networks: Experimental results using LoRa. Paper presented at the VDE ITG-Fachbericht Mobilkommunikation, Osnabruck, Germany, 1-5, 2018.

20. El Chall R, Lahoud S, El Helou M. LoRaWAN network: Radio propagation models and performance evaluation in various environments in Lebanon. IEEE Internet of Things Journal 6(2) (2019) 2366-2378. doi: 10.1109/JIOT.2019.2906838.

21. Yilmaz VS, Bilgin G, Aydin E, Kara A. Miniaturised antenna at sub- $\mathrm{GHz}$ band for industrial remote controllers. IET Microwaves, Antennas \& Propagation 13(9) (2019) 1408-1413. doi: 10.1049/ietmap.2018.5886.

22. SEMTECH LoRa SX1276 Datasheet. Available online (last accessed on 12.11.2020): https://www.semtech.com/products/wireless-rf/ lora-transceivers/sx1276.

23. Bertoni HL. Radio Propagation for Modern Wireless Systems, Prentice-Hall, London, 2000.

24. Rappaport TS. Wireless Communications: Principles and Practice, Prentice-Hall, Upper Saddle River, New Jersey, 2009.

25. Okumura T, Ohmori E, Fukuda K. Field strength and its variability in VHF and UHF land mobile service. Review Electrical Communication Laboratory 16(9-10) (1968) 825-873.

26. Hata, M. Empirical formula for propagation loss in land mobile radio services. IEEE Transactions on Vehicular Technology 29(3) (1980) 317-325. doi: 10.1109/T-VT.1980.23859.

27. COST Action 231. Digital mobile radio towards future generation systems, final report. European Communities (1999) EUR 18957. 